\title{
COVID-19 and physical inactivity: 'Teetering' on the edge of a deadlier pandemic?
}

\section{Sherief Ghozy ${ }^{1}$, Abdelaziz Abdelaal ${ }^{2}$, Jaffer Shah ${ }^{3}$, Kate Elizabeth Parker ${ }^{4 *}$, Sheikh Mohammed Shariful Islam**}

${ }^{1}$ Faculty of Medicine, Mansoura University, Mansoura, Egypt

${ }^{2}$ Faculty of Medicine, Tanta University, Tanta, Egypt

${ }^{3}$ Drexel University College of Medicine, Philadelphia, Pennsylvania, USA

${ }^{4}$ Institute for Physical Activity and Nutrition (IPAN), School of Exercise and Nutrition Sciences, Deakin University, Geelong, Australia

*Equal senior authorship.

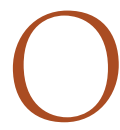
n March 11, 2020, the World Health Organization (WHO) declared COVID-19 as a global pandemic. To limit its spread, most countries recommended social distancing, quarantine and self-isolation, and closure of schools and most businesses. This has resulted in a substantial negative impact on human society regarding health, economy, and lifestyle. In turn, health care policymakers focused on diagnosis and management of COVID-19, as well as the development of effective vaccines. However, the long-term effects of the COVID-19 pandemic on physical activity (PA) and sedentary behaviour (SB) of the world's population, secondary to quarantine, with their consequent burden on global health, have not been given their required attention. We aim to highlight the need

Physical inactivity during the COVID-19 pandemic may lead to a deadlier 'second wave' of ill health and wellbeing long-term. for all physicians to include a prescription of increasing PA and reducing SB for their patients during and following COVID-19, particularly those with known comorbidities.

Physical activity is an essential indicator of health status and has been highlighted as critically important for mitigating the harms of the coronavirus disease-2019 on health and well-being. Quarantine and social distancing measures are impacting the ways in which people can be active and many studies have shown a significant decrease in PA participation concurrent with an increase in SB compared to pre-pandemic levels. For example, Tison et al. [1] conducted a study to determine the daily step count, an indicator of PA, among 455404 individuals from 187 different countries. The mean daily step count reduced by $5.5 \%$ within the first 10 days of pandemic declaration and by $27.3 \%$ within 30 days. This figure is expected to worsen as time goes by. Meyer et al. studied changes in PA and screen-based SB in 3052 US adults from all 50 states. They found a clinically meaningful reduction in PA and increase in sitting and screen time (common indicator of SB) among those in self-isolation, with decreases in PA (-32\%) seen to be worst among those who were previously active [2]. These lifestyle changes were also observed in children. For example, studies conducted with large samples of children and adolescents in the US [3] and Germany [4] saw a reduction in PA and greater engagement in SB during the pandemic compared to earlier periods. These studies highlight dramatic negative changes in $\mathrm{PA}$ and $\mathrm{SB}$, which is concerning given the considerable health risks associated with insufficient PA and excessive SB. 
It is well-documented that regular engagement in physical activities enhances the effectiveness of the immune system [5], which would affect the severity of COVID-19. Therefore, the noticeable increase in physical inactivity and excess sedentary time could potentially lead to the deterioration of infected cases and further promote the spread of the disease. Furthermore, there are serious concerns that the documented short-term deteriorations in PA and increases in SB may become permanently fixed. This may subsequently increase the risk of obesity, diabetes, and cardiovascular diseases, as well as exacerbate the severity of existing conditions [6].

Obesity and PA are linked, with an almost 4-fold increase in the risk of obesity in physically inactive individuals [7]. Obese individuals are also disproportionately affected by COVID-19 infection [8]. It is reported that obesity increases the risk of COVID-19 infection by $46 \%$, hospitalizations by $113 \%$, ICU admission by $74 \%$ and mortality by $48 \%$ [8]. Moreover, obesity is linked with major cardiometabolic disorders, including hypertension, diabetes, and other comorbidities. These comorbidities have been reported to be associated with worse prognosis and serious adverse events in patients with COVID-19, as hypertension and diabetes were documented in $13.34 \%$ and $9.65 \%$ of total COVID-19 cases and $47.90 \%$ and $24.89 \%$ of mortality cases, respectively [9]. These statistics warn us about the current situation and impact of COVID-19 on obesity, secondary to physical inactivity and SB, with the potential of a 'second wave' that would be even worse compared to the early pandemic statistics. Therefore, an increase in PA and reduction in SB should be advocated to avoid any permanent changes in behavior and associated ill health lasting beyond the quarantine duration. For instance, physicians could inform their patients on ways to remain active during times of social distancing and isolation by performing outdoor low-to-moderate intensity PA daily, incorporate

Comprehensive efforts by healthcare professionals, policymakers, educators and community groups are required to increase physical activity and reduce sedentary behavior among the population; during and after the COVID-19 pandemic. stretching and breathing deeply, break up sitting times with PA, standing up, and stretching and avoiding inactivity throughout the day [10]. During the COVID-19 pandemic, there have been substantial increases in the use of remote and telehealth services by physicians. These online services also offer an ideal platform whereby physicians can conduct PA and SB monitoring and adjunct behaviour change approaches to patients with inactive lifestyles when access to in-person services are unavailable. PA promotion interventions can be implemented in primary health care settings. Primary care professionals are well placed to support positive changes in PA and SB since they represent a trusted source of health advice. However, physicians may not be fully equipped to provide appropriate PA counselling, as it is not comprehensively covered in the medical curriculum [11], and counselling time with patients is limited allowing only a few issues to be addressed in a single visit. Thus, there may be limited capacity to assess current activity levels, determine readiness for change or prescribe PA. Despite these limitations, PA promotion interventions in primary health care settings have shown short and long-term effectiveness and should be enforced by primary care commissioners and local government health departments $[12,13]$.

In addition to intrapersonal support mechanisms such as physicians prescribing and supporting their patients to be active, population level initiatives are also required. As proposed by socio-ecological models, lifestyle behaviours are influenced by a broad range of factors and as such, efforts to support and maintain PA require comprehensive and synergistic efforts by policymakers, educators, community groups, health care professionals, and the general population [14]. Such initiatives may include policymakers limiting or prohibiting vehicle traffic at certain times to encourage active travel, sporting organisations and educators harnessing social

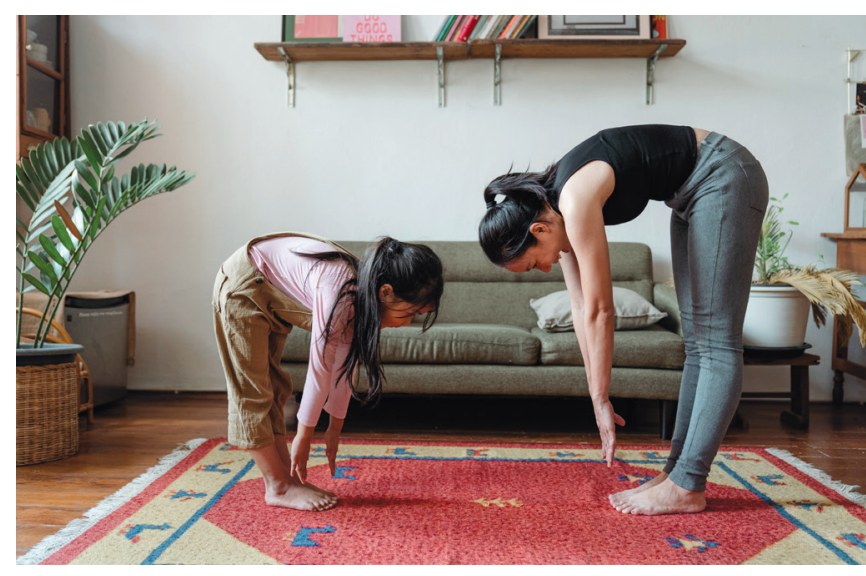

Photo: From https://unsplash.com/. media and online platforms to promote safe, age-appropriate home-based exercises or family activity challenges, and community groups providing information on local walking routes and green spaces to encourage neighbourhood activity. For children and adolescents, enjoyment is a key factor so, creative methods like walking a dog, TikTok dancing, online PA classes, gaming-based PA, and scavenger hunts; should be used. At the community level, parents should be educated about how to maintain the PA of their children at home along with showing children-oriented educational videos at schools and community centres.

Global initiatives for a COVID-19 vaccine are showing promise. However, a major concern is that COVID-19 vaccines would be less effective in obese individuals compared to the general population [8], given the fact that obesity is 
linked to suboptimal vaccine responses for a number of diseases, such as influenza [15] and tetanus [16]. Thus, in addition to population wide policy and strategy implementation, it is critical that physicians include a prescription of PA and a reduction in SB for prevention and management of obesity and associated comorbidities, not only during the pandemic and initial vaccination phases, but also in the long-term.

\section{Funding: None.}

Authorship contributions: SG had the initial concept and AA, JS, KEP, and SMSI contributed to writing and revision of further drafts. KEP and SMSI supervised all steps. All authors contributed and meet the ICMJE criteria for authorship and have read and approved final draft

Competing interests: The authors completed the ICMJE Unified Competing Interest form (available upon request from the corresponding author), and declare no conflicts of interest.

1 Tison GH, Avram R, Kuhar P, Abreau S, Marcus GM, Pletcher MJ, et al. Worldwide Effect of COVID-19 on Physical Activity: A Descriptive Study. Ann Intern Med. 2020;173:767-70. Medline:32598162 doi:10.7326/M20-2665

2 Meyer J, McDowell C, Lansing J, Brower C, Smith L, Tully M, et al. Changes in Physical Activity and Sedentary Behavior in Response to COVID-19 and Their Associations with Mental Health in 3052 US Adults. Int J Environ Res Public Health. 2020;17:6469. Medline:32899495 doi:10.3390/ijerph17186469

3 Dunton GF, Do B, Wang SD. Early effects of the COVID-19 pandemic on physical activity and sedentary behavior in children living in the U.S. BMC Public Health. 2020;20:1351. Medline:32887592 doi:10.1186/s12889-020-09429-3

4 Schmidt SCE, Anedda B, Burchartz A, Eichsteller A, Kolb S, Nigg C, et al. Physical activity and screen time of children and adolescents before and during the COVID-19 lockdown in Germany: a natural experiment. Sci Rep. 2020;10:21780. Medline:33311526 doi:10.1038/s41598-020-78438-4

5 Krüger K, Mooren F-C, Pilat C. The immunomodulatory effects of physical activity. Curr Pharm Des. 2016;22:3730-48. Medline:27000826 doi:10.2174/1381612822666160322145107

6 Mattioli AV, Sciomer S, Cocchi C, Maffei S, Gallina S. Quarantine during COVID-19 outbreak: Changes in diet and physical activity increase the risk of cardiovascular disease. Nutr Metab Cardiovasc Dis. 2020;30:1409-17. Medline:32571612 doi:10.1016/j.numecd.2020.05.020

7 Pietiläinen KH, Kaprio J, Borg P, Plasqui G, Yki-Järvinen H, Kujala UM, et al. Physical inactivity and obesity: a vicious circle. Obesity (Silver Spring). 2008;16:409-14. Medline:18239652 doi:10.1038/oby.2007.72

8 Popkin BM, Du S, Green WD, Beck MA, Algaith T, Herbst CH, et al. Individuals with obesity and COVID-19: A global perspective on the epidemiology and biological relationships. Obes Rev. 2020;21:e13128. Medline:32845580 doi:10.1111/obr.13128

9 Gold MS, Sehayek D, Gabrielli S, Zhang X, McCusker C, Ben-Shoshan M. COVID-19 and comorbidities: a systematic review and meta-analysis. Postgrad Med. 2020;132:749-55. Medline:32573311 doi:10.1080/00325481.2020.1786964

10 Jurak G, Morrison SA, Leskošek B, Kovač M, Hadžić V, Vodičar J, et al. Physical activity recommendations during the coronavirus disease-2019 virus outbreak. J Sport Health Sci. 2020;9:325-7. Medline:32426171 doi:10.1016/j.jshs.2020.05.003

11 Ward M A Survey of Physical Activity in Medical Curricula: A report of the HEPA in Health Care Settings Working Group 2015.

12 Orrow G, Kinmonth A-L, Sanderson S, Sutton S. Effectiveness of physical activity promotion based in primary care: systematic review and meta-analysis of randomised controlled trials. BMJ. 2012;344:e1389. Medline:22451477 doi:10.1136/bmj.e1389

13 Sanchez A, Bully P, Martinez C, Grandes G. Effectiveness of physical activity promotion interventions in primary care: A review of reviews. Prev Med. 2015;76:S56-67. Medline:25263343 doi:10.1016/j.ypmed.2014.09.012

14 Sallis J, Owen N, Fisher E. Ecological Models of Health Behavior. Health Behavior and Health Education. 2008;4.

15 Neidich SD, Green WD, Rebeles J, Karlsson EA, Schultz-Cherry S, Noah TL, et al. Increased risk of influenza among vaccinated adults who are obese. Int J Obes (Lond). 2017;41:1324-30. Medline:28584297 doi:10.1038/ijo.2017.131

16 Eliakim A, Schwindt C, Zaldivar F, Casali P, Cooper DM. Reduced tetanus antibody titers in overweight children. Autoimmunity. 2006;39:137-41. Medline:16698670 doi:10.1080/08916930600597326

\section{Correspondence to:}

Sheikh Mohammed Shariful Islam MBBS, MPH, PhD, FESC Institute for Physical Activity and Nutrition (IPAN)

School of Exercise and Nutrition Sciences, Faculty of Health

Deakin University

Melbourne, VIC 3125

Australia

shariful.islam@deakin.edu.au 